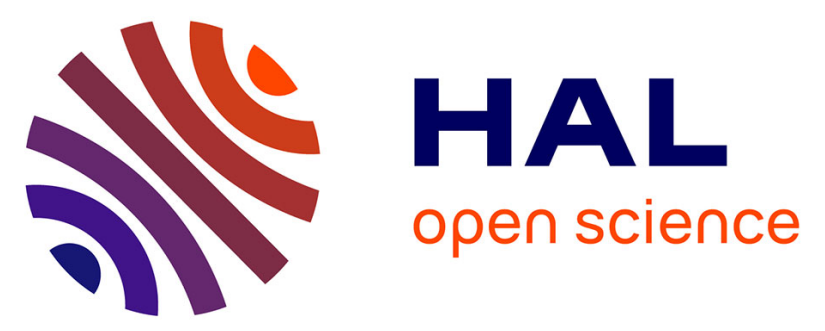

\title{
Possible Artifacts of Fluorescence of Dyes in Concentrated Materials: Comment on "Highly Fluorescent Rhodamine B Nanoparticles Entrapped in Hybrid Glasses"
}

Pierre-André Cazade, Patrice Bordat, Sylvie Blanc, Isabelle Baraille, Ross

Brown

\section{To cite this version:}

Pierre-André Cazade, Patrice Bordat, Sylvie Blanc, Isabelle Baraille, Ross Brown. Possible Artifacts of Fluorescence of Dyes in Concentrated Materials: Comment on "Highly Fluorescent Rhodamine B Nanoparticles Entrapped in Hybrid Glasses". Langmuir, 2008, 24 (5), pp.2252 - 2257. 10.1021/la702291h . hal-03227498

\section{HAL Id: hal-03227498 \\ https: / hal-univ-pau.archives-ouvertes.fr/hal-03227498}

Submitted on 17 May 2021

HAL is a multi-disciplinary open access archive for the deposit and dissemination of scientific research documents, whether they are published or not. The documents may come from teaching and research institutions in France or abroad, or from public or private research centers.
L'archive ouverte pluridisciplinaire HAL, est destinée au dépôt et à la diffusion de documents scientifiques de niveau recherche, publiés ou non, émanant des établissements d'enseignement et de recherche français ou étrangers, des laboratoires publics ou privés. 


\section{Comments}

\section{Possible Artifacts of Fluorescence of Dyes in Concentrated Materials: Comment on "Highly Fluorescent Rhodamine B Nanoparticles Entrapped in Hybrid Glasses"}

\section{Introduction}

Hybrid organic-inorganic materials are attracting much attention due to their potential for a variety of applications as photoactive media, including laser gain media, nonlinear optical devices, photocatalysis, and environmental monitoring. These materials combine the particular photophysical or coloring properties of useful but fragile organic dyes, with the transparency, hardness, and ease of handling of an inorganic host matrix. Often, the dye is encapsulated during sol-gel synthesis of a porous inorganic host material. Molecular organization is of paramount importance for applications, and much effort has been applied to modulating the nanostructure of the organic guest in the mineral host.

Fluorescence spectroscopy naturally occupies a central position in the field, because of its intrinsic sensitivity (down to single molecule level ${ }^{1}$ ) and of the sensitivity of molecular electronic spectra to perturbation by the environment. It is thus an appealing tool for investigating dimerization or clustering of dyes in hybrid materials. In particular, spectral broadening or the growing in of new bands at high dye concentrations is often interpreted as a sign of intermolecular coupling among dyes in dimers, clusters, or crystallites.

However, fluorescence measurements on concentrated samples are susceptible to instrumental artifacts, some of which are quite subtle, and to which we should like to draw attention.

Thus, del Monte et al. recently published an intriguing report on synthesis of highly fluorescent rhodamine B nanoparticles, encapsulated in a transparent silica-based sol-gel glass. ${ }^{2}$ It is indeed difficult to obtain concentrated, modified silicas of high transparency. Their work follows earlier reports on rhodamine B dimers in silica ${ }^{3}$ and similar reports of rhodamine $110 \mathrm{~J}$-dimers, ${ }^{4}$ rhodamine $6 \mathrm{G}$ dimers ${ }^{5}$ rhodamine 19 dimers, ${ }^{6}$ and Nile Red nanoparticles. ${ }^{7}$ Since this pioneering work in synthesis of this type of organically modified silica has justifiably attracted some attention in the literature, and since the main evidence of clustering is interpretation of concentration dependent changes of the electronic spectra, it is important to be sure that these changes are significant.

We show here that all the spectral features ascribed in ref 2 to clustering may also arise from instrumental artifacts. We proceed first in section 3 by an experimental illustration in liquid solutions of comparable optical density to the samples of del Monte et al. A very simple Monte Carlo simulation of light propagation in the absorbing and scattering solids is then applied

(1) Kirstein, J.; Platschek, B.; Jung, C.; Brown, R.; Bein, T.; Bräuchle, C. Nat. Mater. 2007, 6, 303-310.

(2) Gutiérrez, M. C.; Hortigüela, M. J.; Ferrer, M. L.; del Monte, F. Langmuir 2007, 23, 2175-2179.

(3) del Monte, F.; Levy, D. J. Phys. Chem. B 1998, 102, 8036-8041.

(4) del Monte, F.; Levy, D. J. Phys. Chem. B 1999, 103, 8080-8086.

(5) del Monte, F.; MacKenzie, J. D.; Levy, D. Langmuir 2000, 16, 73777382

(6) Ferrer, M. L.; del Monte, F.; Levy, D. Langmuir 2003, 19, 2782-2786.

(7) Ferrer, M. L.; del Monte, F. J. Phys. Chem. B 2005, 109, 80-86. to the xerogels, in section 3.2. It produces qualitatively the same effects as observed, with the same order of magnitude as found by del Monte et al. While the model does not formally "disprove" the presence of molecular clusters, it provides a simpler, consistent explanation of the data in Figures 5 and 7 and Table 1 of ref 2 .

The high optical density (O.D.) of other materials prepared by similar methods ${ }^{3-7}$ and accompanied by similar spectral features ascribed to clustering, leads to concern that artifacts are significant in these results too. On the other hand, the absorbance of samples in ref 8, which do not show the characteristic effects discussed below, is only $<0.03$, consistent with our interpretation.

While we are here specifically concerned by the work in ref 2 , for which detailed experimental information was published, reports of dye clustering in concentrated media are not uncommon, based on spectra showing the marks of the artifacts discussed below, e.g., refs 9-13. It thus seems useful to re-discuss these "classic" but frequently underestimated effects. Indeed, one of us (R.B.) successfully-albeit we now see with hindsight inappropriately-applied exciton theory to literature data showing broadening of diffuse reflectance spectra of dye-impregnated silica powders. ${ }^{14}$ It is only since we sought to experimentally reproduce and generalize the data that we have understood the severity of the fluorescence artifacts, even in very thin films and fine powders.

After a brief reminder on fluorescence artifacts in section 2, section 3 below illustrates these effects with experimental data on concentrated dye solutions, obtained in conditions as similiar as possible to those prevailing in the solid state measurements in ref 2. We then apply Monte Carlo simulation to tracking photons in an absorbing, scattering, and fluorescent material to illustrate how these considerations apply equally to solid samples such as the doped xerogels studied by del Monte et al. We conclude that the concentration-dependent spectral and lifetime data interpreted by them in terms of clustering could just be consequences of excessive optical density of the samples.

\section{Fluorescence Artifacts}

Fluorescence excitation spectra of optically thick (concentrated) samples are prone to intensity distortion when the high optical density causes saturation of the signal at band peaks - essentially all the incident light is absorbed at these wavelengths, so the fluorescence signal stagnates, whereas excitation band tails may continue to grow in intensity as the concentration is increased in the same range. If light scattering is momentarily set aside, and a sample of thickness less than the focal spot size in a spectrometer is considered, the effect is captured in the relation

(8) del Monte, F. J. Mater. Chem. 2001, 11, 1745-1751.

(9) Miller, E. J. Photochem. Photobiol., A: Chem. 2002, 152, 249-257.

(10) Vogel, R.; Meredith, P.; Harvey, M. D.; Rubinsztein-Dunlop, H. Spectrochim. Acta, Part A 2004, 60, 245-249.

(11) De, S.; Das, S.; Girigoswami, A. Spectrochim. Acta, Part A 2005, 61, $1821-1833$

(12) Deshpande, A. V.; Kumar, U. J. Fluoresc. 2006, 16, 679-687.

(13) Anedda, A.; Carbonaro, C. M.; Corpino, R.; Ricci, P. C.; Grandi, S ; Mustarelli, P. C. J. Non-Cryst. Solids 2007, 353, 481-485.

(14) Brown, R.; Lacombe, S.; Cardy, H. Microporous Mesoporous Mater. 2003, 59, 93-103. 


$$
I_{\text {fluo }} \propto I_{\text {ex }}\left\{1-\exp \left[-\ln (10) \epsilon\left(\lambda_{\text {ex }}\right) C l\right]\right\}=I_{\text {ex }}\left(1-10^{-A\left(\lambda_{\text {ex }}\right)}\right)
$$

where $I_{\text {fluo }}$ is the fluorescence signal, proportional to the total light absorbed by a sample of thickness $l$. In eq 2, $\epsilon\left(\lambda_{\text {ex }}\right)$ is the molar extinction coefficient at excitation wavelength $\lambda_{\text {ex }}$ and $C$ is the molar dye concentration. The absorbance is given by $A\left(\lambda_{\text {ex }}\right)$ $=\epsilon\left(\lambda_{\mathrm{ex}}\right) C l$.

The strong nonlinearity of the relation between $C$ and $I_{\text {fluo }}$ at high absorbance or O.D. leads to an artifact, which enhances band tails, so that the apparent spectrum is broadened, an effect that could be mistaken for real broadening due to intermolecular interactions in concentrated solutions. Even weak vibronic bands, hardly noticeable in the excitation spectrum band tail at low concentration, may be promoted to prominent "new" bands in concentrated samples. The other consequence of relation 2 is that the fluorescence intensity, be it the peak value, or that recorded at a constant wavelength or the value integrated over the whole spectrum, grows sublinearly with concentration at higher concentrations and eventually stagnates. The process is related to the familiar "inner filter" phenomenon observed when excitation spectra of concentrated solutions are recorded in $90^{\circ}$ geometry in standard $1 \mathrm{~cm}$ cells: ${ }^{15,16}$ The fluorescence signal at first grows proportionally to the concentration but turns over, eventually decreasing with increasing concentration, because the excitation beam is absorbed at the face of the cell, away from the central part which is imaged by the collection optics onto the detector or monochromator.

The second effect, reabsorption of the electronic origin, affects emission spectra. ${ }^{15,16}$ It occurs when the absorption and emission $0-0$ bands overlap, so that fluorescence emitted in the depths of an optically thick sample may be reabsorbed by it. Re-emission in random directions or nonradiative transitions then lead to a loss of signal in the direction of the collection optics and to decreased fluorescence intensity in the region of spectral overlap of absorption and emission. The resultant loss of signal in the blue wing of the emission spectrum produces an apparent red shift of the $0-0$ band emission maximum, which could be erroneously ascribed to the red shift to be expected from, e.g., the increase of the average electronic polarizability of the medium, or to exciton effects at high dye concentrations or in clusters.

Reabsorption is also well-known to lengthen the apparent fluorescence lifetime, ${ }^{17}$ since every reabsorbed photon is in effect "stored" for on average the fluorescence lifetime before it can be re-emitted and escape from the sample.

\section{Illustration}

3.1. Experimental Illustration of Spectral Effects. del Monte et al. prepared a series of silica xerogels containing glycidoxypropyltrimethoxysilane, with increasing concentrations of rhodamine $\mathrm{B}$ in the starting solution, leading to concentrations of rhodamine $\mathrm{B}$ in the final materials in the range $C=10^{-5}$ $10^{-2} \mathrm{M}$.

The authors characterized the materials by IR spectroscopy, ${ }^{13} \mathrm{C}$ NMR spectroscopy, scanning electron microscopy, and, principally, fluorescence spectroscopy. As in earlier papers, they applied the theory of exciton splitting to interpret modifications of the spectra at higher rhodamine B concentrations, as indicating specific forms of association of the dye in the host matrix, leading

(15) Lakowicz, J. R. Principles offluorescence spectroscopy; Springer: Berlin, 1999.

(16) Valeur, B. Molecular fluorescence. Principles and applications; WileyVCH: Weinheim, 2002.

(17) Becker, W.Advanced time-correlated single photon counting techniques: Springer: Berlin, 2005.
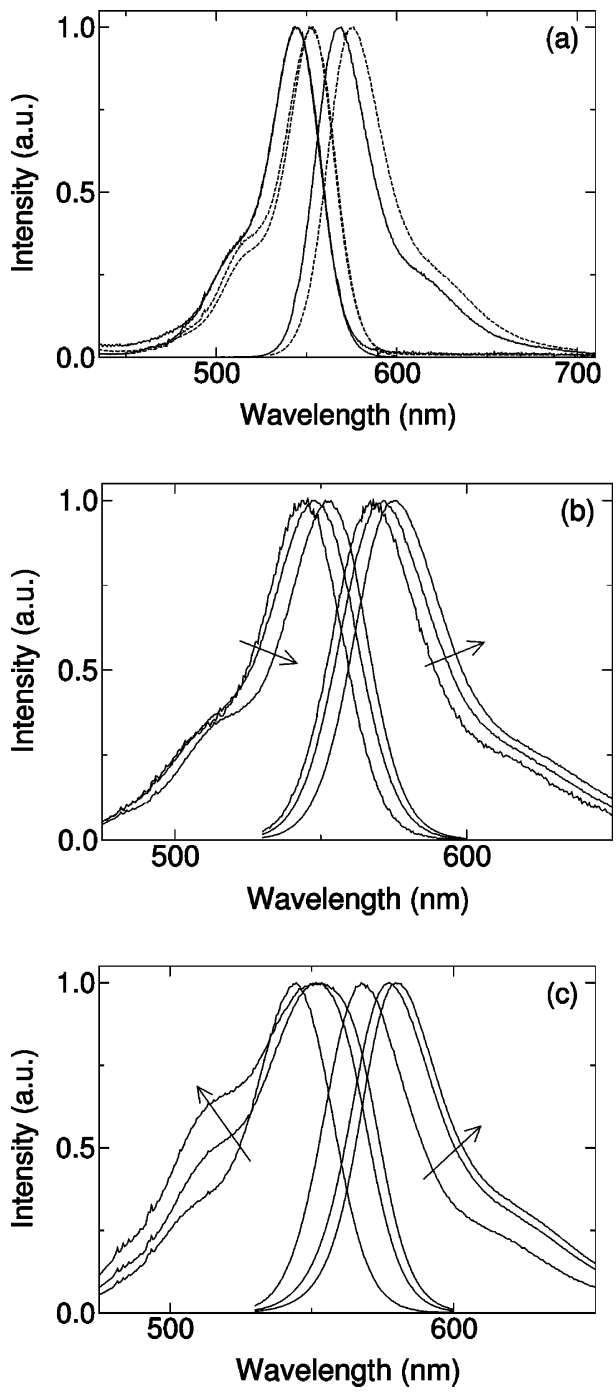

Figure 1. Experimental illustration of fluorescence artifacts in optically thick samples. (a) Reference absorption, excitation (nearly overlaid on the absorption curves), and emission spectra of rhodamine $\mathrm{B}$ in ethanol. Solid curves, $5 \times 10^{-7} \mathrm{M}$ in a $1 \mathrm{~cm}$ cell; dashed curves, $10^{-3} \mathrm{M}$ in a $10 \mu \mathrm{m}$ cell; (b) excitation and emission spectra at concentrations $5 \times 10^{-5}, 5 \times 10^{-4}$, and $10^{-3} \mathrm{M}$ in a $10 \mu \mathrm{m}$ cell; (c) same in a $100 \mu \mathrm{m}$ cell. Arrows in (b) and (c) indicate the order of increasing concentration. All spectra are normalized to unit peak height.

eventually to nanoparticles with "enhanced fluorescence" in the most concentrated samples.

In their work, del Monte et al. pointed out that one should beware of the above artifacts, of concern in fluorescence spectroscopy of concentrated samples. They took the precaution of using a "front-face" setup ${ }^{15,16}$ and ground up samples of their materials, in $0.2 \mathrm{~mm}$ diameter capillaries, rather than the original xerogel monoliths, which were $3 \mathrm{~mm}$ thick. We argue below that these precautions were unfortunately insufficient.

To help grasp the significance of absorption saturation effects, we first present experimental data on rhodamine B in solution in ethanol, recorded by us in cells of different thickness. Figure 1a shows the absorption spectrum of rhodamine B (Exciton, used without further purification) in ethanol (spectroscopic grade, Merck), measured at two concentrations in different cells. Reference absorption spectra (Cary 5 spectrophotometer, double beam mode) and fluorescence excitation and emission spectra (Edinburgh FS900, 90 geometry) were recorded at $1 \mathrm{~nm}$ resolution for a solution at a concentration of $5 \times 10^{-7} \mathrm{M}$ in a 
$1 \mathrm{~cm}$ quartz cell. Emission spectra were recorded here at excitation wavelength $520 \mathrm{~nm}$ and excitation spectra at emission wavelength $620 \mathrm{~nm}$.

The absorption spectrum peaks at $\lambda_{\max }=544 \mathrm{~nm}$, where we find an extinction coefficient of $\epsilon_{\max }=108000 \mathrm{M}^{-1} \mathrm{~cm}^{-1}$, close to the value reported previously for the monomer of rhodamine $\mathrm{B}$ in ethanol, by Selwyn and Steinfeld. ${ }^{18}$ In agreement with this, we therefore assign the band at $544 \mathrm{~nm}$ to the $0-0$ transition and the shoulders in our spectra at very low concentration to vibronic bands at (in our case) $\lambda \approx 520$ and (barely visible) $475 \mathrm{~nm}$. This attribution is consistent with the mirror symmetry of the shoulder in the emission spectrum. At higher concentrations, an isosbestic point reported in ref 18 was interpreted as indicating equilibrium between monomers and dimers in solution. It was accompanied there by a red shift of the absorption maximum, also present in our data.

In what follows, the absorption of the vibronic bands and hence the fluorescence signal derived from them, will saturate at higher concentrations than for the $0-0$ band, giving the impression of concentration-dependent "band splitting". We show below that a similar effect is predicted in solid samples, explaining the apparent splitting reported in Figure 7 of del Monte et al. ${ }^{2}$ There will also be concomitant broadening of the red wing of the $0-0$ band.

The close similarity of the absorption and excitation spectra, which are practically indistinguishable in Figure 1a, confirms the absence of artifacts in this case (peak absorbance $\sim 0.05$ ). Figure 1a also shows the normalized spectra of a $10^{-3} \mathrm{M}$ solution in a $10 \mu \mathrm{m}$ cell (Hellma). The shoulder at ca. $520 \mathrm{~nm}$ is slightly enhanced in the excitation spectrum compared to the absorption spectrum, partly because the O.D. is now on the order of 0.1 . Nonetheless, the general coincidence of the absorption and excitation spectra shows that the sample is still optically thin.

Figure 1b,c illustrates the effects of the O.D. on spectra in ethanol at concentrations in the range $5 \times 10^{-5}-10^{-3} \mathrm{M}$, recorded in quartz cells of thickness 10 and $100 \mu \mathrm{m}$ set at approximately $45^{\circ}$ to the excitation and emission collection directions. The thicknesses and concentrations are chosen to be comparable to those of the powdered doped silicas examined by del Monte et al. Discrepancies between the absorption and excitation spectra increase with concentration, as saturation of the absorption in the $100 \mu \mathrm{m}$ cell causes broadening of all excitation lines and enhances the vibronic bands compared to the $0-0$ transition, similar to the behavior of the doped silicas in Figure 7 of ref 2, and giving the impression of the growing in of new excitation bands. In extreme cases, such effects can actually invert the sequence of maxima and minima in electronic or vibronic bands (see, for example, ref 11) or the excitation spectra of a pyrenedoped silica gel in ref 9 compared to the absorption spectrum in ref 19.

Figure $1 \mathrm{c}$ also illustrates how reabsorption of the $0-0$ transition in the emission spectra causes an apparent red shift of the emission maximum in the thicker sample, similar to that reported in ref 2. In our example solutions, the red shift of the emission spectrum between the dilute and the concentrated samples is $12 \mathrm{~nm}$ in the thick cell compared to the intrinsic value, $7 \mathrm{~nm}$ measured in the thin cell as in Figure 1a.

Except for overall wavelength shifts, the spectra in the reference solution in ethanol are very similar to those of xerogel RBG1, Figure 1 of ref 2 . In order to illustrate by simulation the effect of concentration artifacts in the solid samples, we therefore use

(18) Selwyn, J. E.; Steinfeld, J. I. J. Phys. Chem. 1972, 76, 762-774.

(19) Du, H.; Fuh, R. C. A.; Li, J.; Corkan, L. A.; Lindsey, J. S. Photochem. Photobiol. 1998, 68, 141-142.

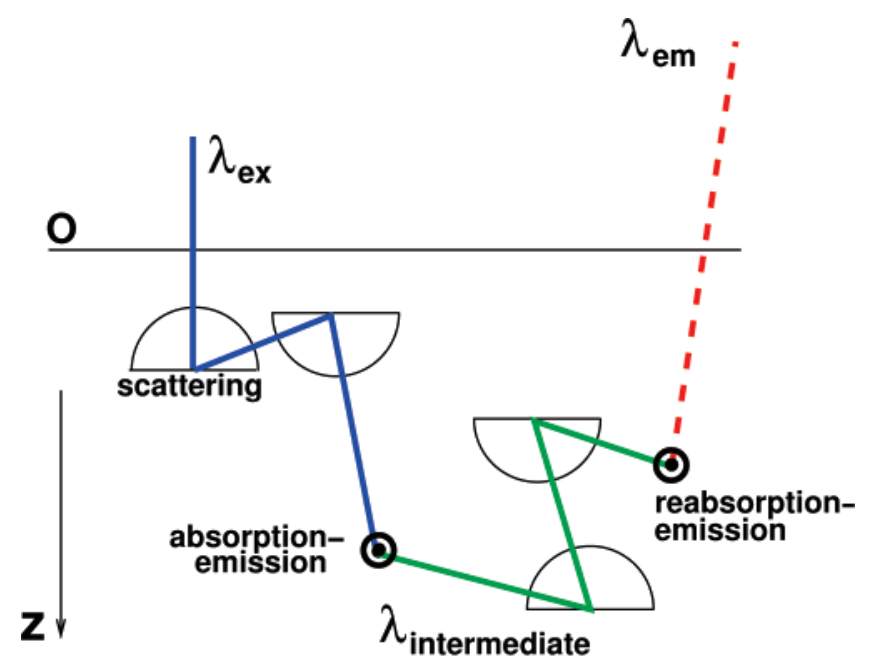

H

Figure 2. Illustration of the Monte Carlo model. This particular photon is scattered twice, absorbed, and emitted in the overlap of the absorption and emission bands; scattered and finally re-absorbed and emitted in the red, escaping from the sample. Sample response is built up by sampling millions of such tracks drawn with probabilities determined by to the photophysical properties of the materials.

these solution spectra, after shifting them to the same peak wavelengths as in xerogel RBG1 $(580 \mathrm{~nm}$ for the excitation spectrum, 592 for the emission spectrum).

3.2. Simulation of Spectral Artifacts in Optically Thick Solid Samples. In practice, one must also take account of the spectral distortion introduced by scattering in powder samples such as used by del Monte et al. Scattering is a complex problem that can be taken into account at varying levels of sophistication. One of the simplest approaches is the Kubelka-Munk two-flux theory of diffuse reflectance, ${ }^{20}$ which can be readily extended to include reabsorption and fluorescence. ${ }^{21,22}$ Other more elaborate possibilities of analysis are multiflux models and more generally the full radiative transfer equations, ${ }^{23}$ diffusion theory of light scattering, ${ }^{24}$ and Monte Carlo simulation. ${ }^{25,26}$ A full calculation requires information on the phase distribution (angular distribution of scattered light). ${ }^{27}$ In the absence of specific information on the present xerogel powder particles, we present here a simple Monte Carlo model assuming predominantly back scattering, justified by the high albedo of ground silica powders. Compared to the steady-state flux equations, Monte Carlo simulation has the added advantage of providing the temporal profile of the response (apparent fluorescence decay).

We calculate the response of a homogeneous slab of material (see Figure 2) in which the actual absorption and scattering of the inhomogeneous packed powder are represented by two numbers, a specific absorption coeffecient $k(\lambda)=\epsilon(\lambda) C$ and a scattering coefficient $s(\lambda)$. These quantities are homogeneous with inverse lengths: $1 / k(\lambda)$ and $1 / s(\lambda)$ are mean free paths before absorption or scattering for photons at wavelength $\lambda$.

(20) Kubelka, P.; Munk, F. Z. Technische Physik 1931, 12, 593-601.

(21) Oelkrug, D.; Kortum, G. Z. Phys. Chem. 1968, 58, 181-188.

(22) Lagorio, M. G.; Dicelio, L. E.; Litter, M. I.; San Roman, E. J. Chem. Soc., Faraday Trans. 1998, 94, 419-425.

(23) Chandrasekhar, S. Radiative Transfer; Dover: New York, 1960.

(24) Illarramendi, M. A.; Aramburu, I.; Fernandez, J.; Balda, R.; Williams,

S. N.; Adegoke, J. A.; Noginov, M. A. J. Opt. Soc. Am. B 2007, 24, 43-48.

(25) Wang, L.; Jacques, S. L. J. Opt. Soc. Am. A 1993, 10, 1746.

(26) Hehlen, M. P. J. Opt. Soc. Am. B 1997, 14, 1312-1318.

(27) Hapke, B. Theory of reflectance and emittance spectroscopy; C. U. P.: Cambridge, 1993. 
The slab, thickness $H$, lies horizontally in the $x y$ plane. The positive $z$ direction is taken into the slab. Photons of wavelength $\lambda_{\text {ex }}$ impinge normally on the "front" face $(z=0)$ at time $t=0$. On entering the material, photons undergo a sequence of scattering or absorption and re-emission events determined by a Markov process (no spatial or temporal memory) until either they emerge at the front or the back face of the slab or they disappear by a nonradiative process, with yield $1-Q_{\mathrm{F}}$, where $Q_{\mathrm{F}}$ is the fluorescence quantum yield. Random sequences of these events are generated with the minimal process algorithm (see Supporting Information). This semi-infinite model applies to typical spectrometer geometries provided that the $x, y$ spread of photon paths does not exceed the size of the conjugate images of the spectrometer slits, a condition met in the present simulations. Generalization is possible by Monte Carlo ray tracing including specific sample and spectrometer geometrical parameters, focal lengths of optical elements, and so forth.

$Q_{\mathrm{F}}$ and $s(\lambda)$ are assumed for our purposes of illustration to be independent of the wavelength, but generalization to variable $Q_{\mathrm{F}}$ and $s$ is trivial. In the absence of specific information on the scattering phase distribution, we assume isotropic back scattering into the hemisphere with horizontal base plane centered on the scattering site and pole on the same side of the scattering site as the incident photon; see Figure 2. Photons emerging from the front face of the slab represent the observed diffuse reflectance and fluorescence signal and are binned with respect to wavelength and time to produce a fluorescence spectrum or a fluorescence decay. By repeating the simulations with varying $\lambda_{\mathrm{ex}}$, an excitation spectrum can be built up. The algorithm described in detail in the Supporting Information was coded in Fortran 90. From $10^{7}$ (high $C$ ) to $10^{8}$ (low $C$ ), photons were traced through the sample to obtain the level of statistical noise apparent in the figures below, requiring a few tens of hours of CPU time on a recent 64 bit processor.

The model requires specification of the photophysical properties of the material. As mentioned above, the absorption and fluorescence spectra are approximated by those obtained in solution in ethanol, shifted to have the same peak positions as the corresponding spectra in the most dilute xerogel samples. The fluorescence quantum yield was estimated in ref 2 to be $Q_{\mathrm{F}}$ $=0.9$. We estimated the radiative decay rate from the absorption and emission spectra and the Strickler-Berg relation, ${ }^{28}$ obtaining a value of $2.9 \mathrm{~ns}$, surprisingly rather less than the total fluorescence lifetime $4 \mathrm{~ns}$ in dilute samples obtained in ref 2 . With the accompanying fluorescence quantum yield of 0.9 , one would expect the fluorescence lifetime to be slightly shorter than the radiative lifetime. For the purposes of illustration, we have nonetheless adopted an intermediate "intrinsic" fluorescence lifetime of $3.5 \mathrm{~ns}$.

Apart from a trivial overall scaling of intensities, $s$ is thus the only adjustable parameter in the model. It will be found possible to reproduce all the experimental data with a single value, $s=$ $2000 \mathrm{~cm}^{-1}$. This is to be interpreted as an order of magnitude, since fine details of the simulated results should and do depend somewhat on the assumed phase distribution.

Figure 3 shows the results of the simulations, which qualitatively and quantitatively reproduce the spectral deformations in Figure 7 of ref 2. The model reproduces the apparent broadening of the $0-0$ band accompanied by the relative rise of the vibronic bands in the blue wing of the excitation spectra. There is slightly more vibronic structure in the spectra of the dilute solid sample, Figure 1 of ref 2, than in the reference spectra in ethanol used in the simulation, so that a second "new" band

(28) Strickler, S. J.; Berg, R. A. J. Chem. Phys. 1962, 37, 814-822.
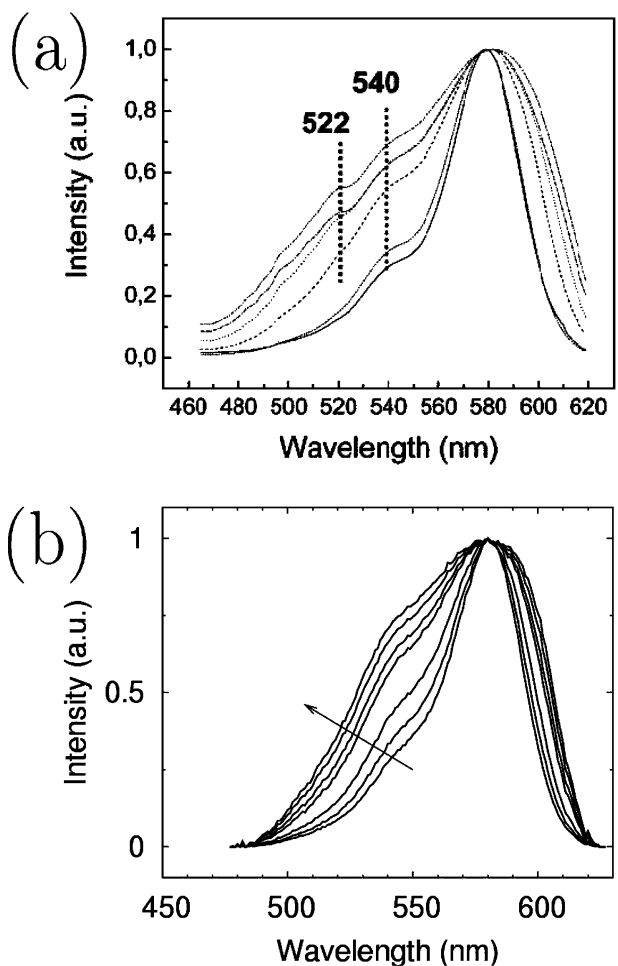

Figure 3. (a) Figure 7 of ref 2, showing excitation spectra of powdered, rhodamine B-doped silica glasses in the concentration range $\left(5.5 \times 10^{-5}\right)-\left(1.1 \times 10^{-2}\right) \mathrm{M}$, suggested in ref 2 to indicate specific clusters of dye molecules. (b) Simulation of the same data with the Monte Carlo model, including the effects of scattering, absorption, fluorescence, and reabsorption in concentrated but homogeneous solid solutions. The arrow indicates increasing concentration. The reference spectra used in the simulation are from a dilute solution in ethanol, Figure 1, shifted to the same peak wavelengths as in the dilute xerogel. All changes in the simulated spectra are due to fluorescence artifacts discussed in the text.

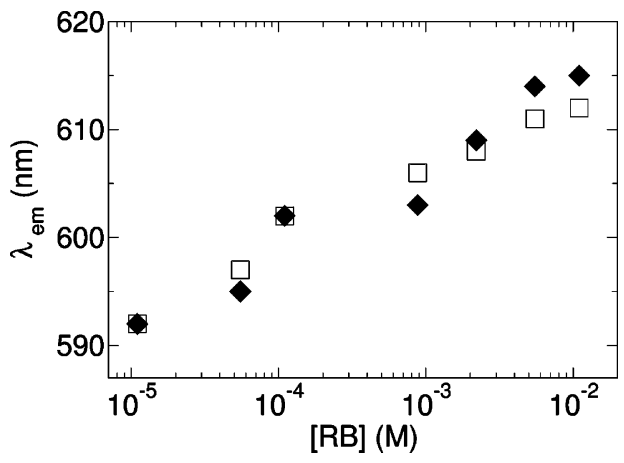

Figure 4. Wavelength of the emission maximum of the powdered dye-doped glass. $\bullet$, from Table 1 , ref 2 , interpreted there as an effect of intermolecular coupling; $\square$, simulated here, see text. The red shift of the maximum in the simulated data is due to reabsorption of the electronic origin in concentrated samples.

appears in the spectra of the concentrated solid samples, but is absent in the simulations. However, we can nonetheless conclude that the evolution of the spectra discussed in ref 2 in terms of exciton bands and aggregates of rhodamine B is also very well described in terms of artifacts coming from the optical setup. Similar deformations that occur in diffuse reflectance spectra of concentrated fluorescent samples ${ }^{29}$ may be relevant in refs $3-7$. Figure 4 shows the simulated concentration dependence of the wavelength of the emission maximum. As the concentration

(29) Mirenda, M.; Lagorio, M. G.; San Roman, E. Langmuir 2004, 20, 36903697. 
(a)

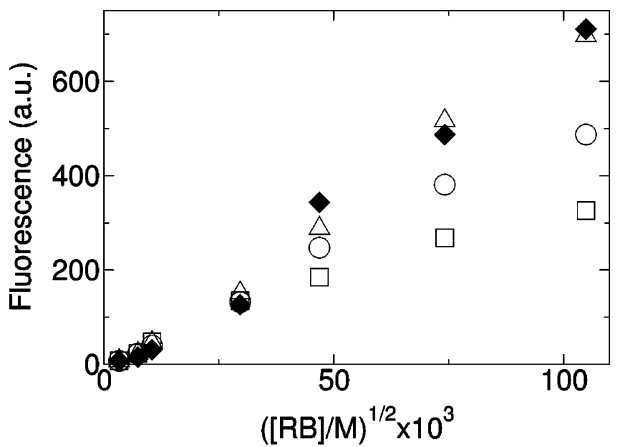

(b)

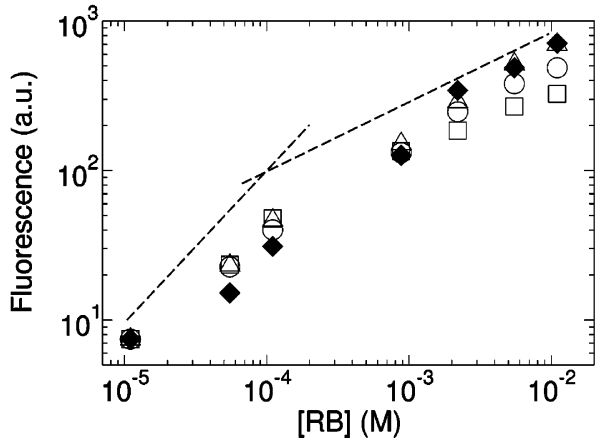

Figure 5. Fluorescence intensity vs concentration in doped xerogel powder samples. (a) Intensity is plotted vs the square root of the rhodamine B concentration. Open symbols, simulated with the Monte Carlo model (see text), same conditions as in Figure 3 and ref 2; $\triangle$, emission intensity in the red wing of the fluorescence spectrum; $\bigcirc$, integrated fluorescence intensity; $\square$, peak fluorescence intensity; $\checkmark$, data redrawn from ref 2 ; (b) $\log -\log$ plot of the simulated fluorescence intensity vs concentration. Saturation of the absorption leads to the overall curvature of the plots. Reabsorption of the electronic origin leads to the stronger curvature of the plot of the peak emission intensity. The dashed lines are guides for the eye, with slopes 1 and $1 / 2$.

increases, reabsorption of the electronic origin causes the maximum to shift to the red, accounting quite well for both the trend and the magnitude of the experimental data.

3.3. Fluorescence Intensity Artifacts. The absorption saturation effect also explains why the fluorescence intensity appears to grow as the square root of the dye concentration in Figure 5 of ref 2. The concentration dependence of the apparent fluorescence intensity can be deduced from the above simulations; see Figure 5. The intensity scaling factor for comparison with the data of ref 2 was determined with respect to the most dilute sample. The concentration dependence of the intensity determined with the same value of $s$ is qualitatively and by order of magnitude in agreement with the experimental data.

One finds that this regime is in fact just part of a saturation curve in which intensity is at first proportional to the concentration, then saturates, giving the appearance of square root behavior at intermediate concentrations, and would finally tend to a constant value at high concentrations. The severity of the effect depends on how one defines the fluorescence intensity. The integrated intensity and above all the peak fluorescence intensity in the $0-0$ band are more sensitive to artifacts, because both absorption saturation and reabsorption of the electronic origin come into play in these cases; see Figure 5b. The variation of the fluorescence intensity is thus sublinear with the dye concentration and there is no reason to assume enhanced fluorescence of the material by molecular clustering compared to what would be obtained with a homogeneous dispersion of molecules. Compared to the

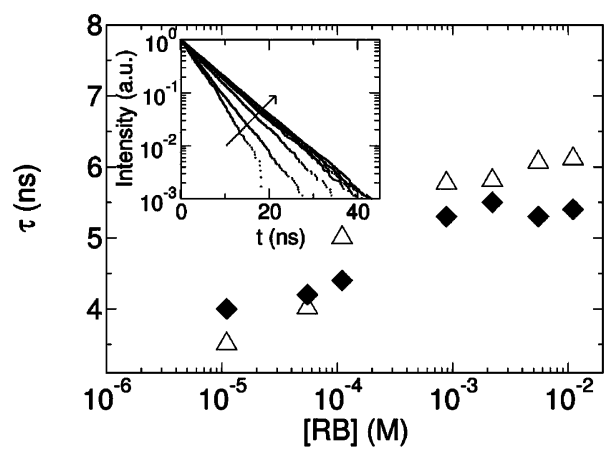

Figure 6. Dependence of the apparent fluorescence lifetime on the concentration of dye in the xerogels. $\bullet$, data from Table 1 , ref 2 , interpreted there as an excitonic effect in molecular clusters; $\Delta$, simulated here without clustering, showing the effect of the light trapping artifact in the more concentrated samples. The inset shows that the simulated decays remain close to exponential. Concentration increases in the direction of the arrow.

prediction of our simple model, two papers on related materials ${ }^{30,6}$ report turning over of the fluorescence intensity as concentration increases. However, these data should be approached with caution, since non-monotonic variation of fluorescence intensity with concentration in optically thick samples is a known artifact. ${ }^{16}$

3.4. Lifetime Artifacts. del Monte et al. reported increased fluorescence lifetimes (about 35\%) between their most dilute and most concentrated samples. ${ }^{2}$ Similar increases are present in the reports on dimers of rhodamine $\mathrm{B},{ }^{3}$ rhodamine $110,{ }^{4}$ rhodamine $6 \mathrm{G},{ }^{5}$ rhodamine $19,{ }^{6}$ and on Nile red nanoparticles. ${ }^{7}$ Consistent with our view of the spectral deformations, we propose that this is just the classical radiation trapping effect ${ }^{26,31,32}$ when the absorption and emission spectra overlap in concentrated samples, leading to repeated reabsorption and re-emission in the electronic origin. ${ }^{17}$ To a first approximation, the apparent decay is expected to remain monoexponential with a lifetime $\tau_{\text {app }}$ related to the true fluorescence lifetime $\tau_{\mathrm{F}}$ by

$$
\tau_{\text {app }} \sim \frac{\tau_{\mathrm{F}}}{1-\phi Q_{\mathrm{F}}}
$$

where $\phi$ is the fraction of light reabsorbed by the sample. ${ }^{31,33}$ By binning the time of emergence of photons from the front face of the slab in the simulations, we can determine the apparent fluorescence decay in the model. For these calculations, we assumed thicker samples $(1 \mathrm{~mm})$, consistent with the experimental procedure of del Monte et al.

Consistent with eq 1 , the apparent fluorescence decay remains closely (though not exactly) exponential at all concentrations examined; see the sample decays in the inset of Figure 6. The apparent lifetime at each concentration was determined from a least-squares fit of a monoexeponential decay to the simulated data. Light trapping is significant in the more concentrated samples, leading to a substantial increase of the apparent lifetime, consistent qualitatively and semiquantitatively with the experimental data; see Figure 6. It is thus unnecessary to appeal to exciton effects to explain the lengthening of the experimental fluorescence decays.

\section{Discussion}

Gutiérrez et al. reported highly fluorescent dye nanoparticles in silica xerogels in ref 2. In our opinion, identification of the bright spots in the SEM image, Figure 6 of their paper, as highly

(30) del Monte, F.; Ferrer, M. L.; Levy, D. Langmuir 2001, 11, 4812-4817.

(31) Birks, J. B. Phys. Rev. 1954, 94, 1567-1573.

(32) Guy, S. Phys. Rev. B 2006, 73, 144101 pages 1-8.

(33) Caird, J. A.; Ramponi, A. J.; Staver, P. R. J. Opt. Soc. Am. B 1991, 8 , 1391-1403. 
fluorescent dye nanoparticles is not sufficiently supported by the spectroscopic evidence, since known artifacts are shown here to lead to data deformations comparable in nature and magnitude to the concentration-dependent features in the recorded data. Illustrative experimental data on concentrated solutions of rhodamine $\mathrm{B}$ and simulations of light propagation in the conditions of high optical density in their paper lead us to question their interpretation. The spectroscopic data in their paper can be explained consistently by artifacts of saturation of optical absorption, reabsorption of the electronic origin, and light trapping, due to the high optical density of the samples employed by these authors.

Concerning concentrated samples in general, we should like to make two further points. First, when working at such high concentrations, consideration should be given to Förster energy transfer. ${ }^{15,16}$ Even assuming a random distribution of dyes, the average nearest-neighbor distance at concentration $\rho$ (molecules per unit volume) is (see Supporting Information) $\bar{r}=\Gamma(1 / 3) /$ $(4 \pi \rho / 3)^{1 / 3}$. This is $\sim 30 \AA$ for the most concentrated xerogel $\left(10^{-2} \mathrm{M}\right)$, well within the range of Förster transfer, for which typical transfer radii (where the transfer rate is the same as the monomolecular decay rate) are 10-50 A. Förster transfer may influence the emission spectrum by energy transfer to low-lying states of two kinds, low-lying monomer states in the inhomogeneous emission band and dimer trap states. ${ }^{34}$ The importance of Förster transfer in the emission spectrum depends in part on the ratio of the spectral broadening (be it inhomogeneous or due to clusters) to the thermal energy, ${ }^{35,36}$ being most significant at low temperatures, where it would induce a red spectral shift. In the present xerogels at room temperature, the spectral width is $\sim 15 \mathrm{~nm} \approx 240 \mathrm{~cm}^{-1}$ half width at half-maximum for the excitation spectrum of RBG1, leading us to expect that this effect is small or negligible.

Second, two qualitative features of the interpretation of del Monte et al. are surprising in the context of clusters. First, clustering is usually associated with a decrease of the fluorescence yield, due to quenching by low-lying excited states reached via energy transfer. ${ }^{15} \mathrm{~A}$ further difficulty of the assumption of clusters is that whereas Selwyn and Steinfeld ${ }^{18}$ show, and we confirm, that the absorption peak is concentration dependent in ethanol, the excitation peak in the silica glasses of ref 2 is not concentrationdependent, in agreement with the simulation of the artifacts, which shows just rounding of the peak. It is unusual that broadening by intermolecular interactions should not be accompanied by some average spectral shift. ${ }^{37}$

(34) Bojarski, P.; Kułak, L.; Grajek, H.;Żurkowski, G.; Kamińska, A.; Kuliński, B.; Borjarski, C. Biochim. Biophys. Acta 2003, 1619, 201-208.

(35) Movaghar, B.; Ries, B.; Grünewald, M. Phys. Rev. B 1986, 34, 55745582.

(36) Madigan, C.; Bulovic, V. Phys. Rev. Lett. 2006, 96, 046404.

(37) Laird, B. B.; Skinner, J. L. J. Chem. Phys. 1989, 90, 3880-3881.
The nature of the bright spots in the SEM image thus remains an open question. From order of magnitude estimations of the optical densities, we suggest that similar concentration dependencies of spectral data in refs $3-7$ could be largely due to the same causes. The limiting O.D.'s in these references may be estimated from the sample thicknesses, molar extinction coefficients, and concentrations to be in the following order: 3, 3, $6,0.3,0.4$. It is $\sim 20$ in the present rhodamine B doped xerogels. One should thus be cautious in attributing these spectral features to fluorescent dye aggregates or nanoparticles. The present artifacts may produce an apparent spectral broadening or even the relative growth of band tails to the point of giving the impression of new bands. They also lengthen the apparent fluorescence lifetime. Whenever possible, it is advisable to record spectra from different thicknesses of a sample in order to help distinguish such effects from genuine spectral broadening due to intermolecular coupling.

In closing, we note that a convenient method to measure highly fluorescent powders is to examine as much as will adhere when gently deposited on transparent sticky tape and the excess shaken off. This trick works quite well for excitation in the visible spectrum, but attention should be paid to fluorescence of the tape under UV excitation. Yet, even this method is unlikely to suffice in the present case, since the O.D. of even a $10 \mu \mathrm{m}$ layer of silica doped with rhodamine $\mathrm{B}$ at $10^{-2} \mathrm{M}$ is on the order of unity.

It remains important to study concentrated hybrid materials. Schemes have been proposed to evaluate and compensate for inner filter effects in fluorescence measurements on concentrated solutions. ${ }^{38}$ They are not readily applicable to scattering materials or thin films. Monte Carlo simulation of light propagation in a specific sample and instrument geometries may prove to be a useful way to allow for fluorescence artifacts in such cases.

Acknowledgment. Part of the simulations were performed on the machines of the M3PEC consortium, Universite de Bordeaux 1.

Supporting Information Available: Monte Carlo method. This material is available free of charge via the Internet at http:// pubs.acs.org.

Pierre-André Cazade, Patrice Bordat, Sylvie Blanc, Isabelle Baraille, and Ross Brown*

Institut pluridisciplinaire de recherche sur l'environnement et les matériaux, umr 5254 du C.N.R.S. et de l'Université de

Pau et des pays de l'Adour, Hélioparc Pau-Pyrénées, 2, avenue Pierre Angot, 64053 Pau Cedex 9, France

Received July 28, 2007

In Final Form: November 16, 2007

LA702291H

(38) Pagano, T. E.; Kenny, J. E. Proc. SPIE 1999, 3856, 289-297. 